Extrême-Orient Extrême-Occident

\section{Extrême-Orient Extrême-Occident}

38 | 2014

La Guerre en perspective : histoire et culture militaire en Chine

\title{
Guerres asymétriques : l'orientalisme militaire contre la Voie de la guerre en Occident
}

Asymmetrical Wars: Military Orientalism against the Way of War in the West

\section{Beatrice Heuser et Patrick Porter}

\section{OpenEdition}

\section{Journals}

Édition électronique

URL : http://journals.openedition.org/extremeorient/379

DOI : 10.4000/extremeorient.379

ISSN : 2108-7105

Éditeur

Presses universitaires de Vincennes

Édition imprimée

Date de publication : 1 novembre 2014

Pagination : 207-218

ISBN : 978-2-84292-414-0

ISSN : 0754-5010

Référence électronique

Beatrice Heuser et Patrick Porter, « Guerres asymétriques : I'orientalisme militaire contre la Voie de la guerre en Occident », Extrême-Orient Extrême-Occident [En ligne], 38 | 2014, mis en ligne le 01 janvier 2017, consulté le 06 mai 2019. URL : http://journals.openedition.org/extremeorient/379 ; DOI :

10.4000/extremeorient.379 


\title{
Guerres asymétriques : l'orientalisme militaire contre la Voie de la guerre en Occident
}

\author{
Beatrice Heuser et Patrick Porter
}

La façon dont une société fait la guerre est souvent vue et présentée comme un reflet de ses valeurs ${ }^{1}$. Depuis l'Antiquité on trouve des auteurs qui pensent pouvoir établir une différence entre la façon dont «nous » faisons la guerre, par opposition à celle qui caractérise les ennemis. Les auteurs parlent des barbares, des bandits, des rebelles, et voient leur façon de faire la guerre comme entachée d'irrégularités. Déjà au Xv $v^{\mathrm{e}}$ siècle avant J.-C., un roi hittite, Mursilis, se plaignait des attaques en pleine nuit par ses adversaires, qu'il estimait contraires aux règles de la guerre ${ }^{2}$. À la période postérieure, l'Antiquité grecque déborde d'exemples de conduite de guerre différant d'un peuple à l'autre. Hérodote souligne ainsi la façon particulière qu'eurent les Scythes de s'attaquer au roi perse Darius qui leur imposait son règne ${ }^{3}$.

Selon Edward Saïd, l'héritage littéraire de l'orientalisme commence avec les guerres entre les Grecs et les Perses. Eschyle fit jouer Les Perses à Athènes en 472 avant J.-C., huit ans après les batailles de Salamine, Platée et Mycale au cours desquelles furent repoussées les tentatives du roi perse Xerxès de conquérir la Grèce. La résistance du peuple grec, et surtout la bataille épique des 300 Spartiates sous Léonide contre les Perses aux Thermopyles, allait devenir le mythe par excellence de leur lutte pour la liberté contre la figure menaçante de l'autre. Les 300 Spartiates qui se sacrifièrent, dans une sorte d' «accomplissement suprêmement grec» sont devenus un symbole pour l'Occident tout entier ${ }^{4}$.

1. Heuser (1998), introduction.

2. Laqueur (1977): 3 .

3. Ellis (1975): p. 15; Hahlweg (1968): 25.

4. Rhodes (2007): 34 . 
Dans Les Perses, Eschyle oppose les vertus grecques aux vices perses, anticipant par là les tropes «orientalistes» ${ }^{5}$. Les hoplites grecs avec leurs boucliers lourds et leurs lances se battent à proximité, honnêtement, tandis que les archers perses combattent à distance. Un peuple libre par nature se bat contre un peuple d'esclaves par nature. Les Perses sont des «hommes dangereux, trop nombreux», qui veulent «imposer fermement aux Grecs le joug de l'esclavage». La Perse, ou l'Orient, gouvernés par un tyran, baignent dans la luxure. Xerxès, le despote, viole l'ordre naturel lorsque, en représentant une puissance terrestre, il prétend lancer un défi aux Grecs par la mer ${ }^{6}$. Notons à cet égard qu'Eschyle s'abstient de mentionner les Grecs de l'Asie mineure, les Ioniens, employés par Xerxès parmi ses propres forces.

Chez les Romains aussi, il y avait une claire conscience des différentes manières de faire la guerre. Dans sa Guerre des Gaules, César nous a laissé de nombreuses preuves de telles asymétries entre la façon de se battre de ses légions et les tactiques et stratégies des Gaulois et des Bretons. Le poète Lucain dans la Pharsale propose une caractérisation des différents peuples, fondée sur la distinction entre les régions et les façons de faire la guerre ${ }^{7}$. Le choc de la défaite des légions romaines par des Chérusques sous Arminius dans une embuscade gigantesque au sein de la forêt de Teutobourg en 9 après J.-C. marqua profondément les Romains. Tacite à son tour a parlé de la façon très particulière qu'avaient eue les Numides de résister aux Romains en Afrique du Nord, pour ne rien dire des incursions et des pillages des cavaliers scythes aux armes légères au cours des siècles suivants.

Il n'est donc guère surprenant que ce soit un classiciste, Victor David Hanson, qui ait le premier extrapolé de ses connaissances de l'histoire militaire en Grèce antique l'idée d'une continuité en Occident dans la façon de faire la guerre, une façon honnête, directe, supposant une confrontation face-à-face sur le champ de bataille, par opposition avec une façon indirecte, malhonnête, recourant à la ruse, aux embuscades, aux attaques surprises ${ }^{8}$. En réalité, la distinction n'est pas si nette, même s'il y avait bien en Europe des peuples qui se battaient «autrement». Hanson a été fortement critiqué par ceux qui n'occultent pas, contrairement à lui, les époques au cours desquelles la façon prédominante de faire la guerre, même en Europe, ne consistait pas seulement à livrer bataille mais à assiéger, à recourir à de grandes charges à cheval en

5. Eschyle (472 BCE): lignes 52, 71, 399, 658.

6. Goldhill (1988): 190.

7. Lucain (av. $65 \mathrm{CE}$ ) : I.455 sq.

8. Hanson (1989). 
s'autorisant le pillage et la destruction des forteresses, des villes, des villages et des champs, comme à employer de façon constante à la ruse ${ }^{9}$.

Au cours du Moyen Âge, les Occidentaux voyaient les Slaves comme particulièrement barbares dans leur façon de faire la guerre, à l'image de ces peuplades situées plus à l'Ouest, les Celtes de Grande Bretagne. Giraldus Cambrensis (ou Giraud le Cambrien), le grand ecclésiastique et lettré gallois du XII ${ }^{e}$ siècle, lui-même fils de princesse galloise et de seigneur normand, nous a laissé son témoignage sur la manière qu'avaient les Gallois de faire la guerre, qui contrastait avec celle des Normands caractérisée par une stratégie de domination et l'apaisement de la population locale par l'intermédiaire des châteaux-forts et du commerce.

Les Gallois, écrit-il, sont légers et agiles. [...] Ils n'hésitent pas à lutter sans protection contre des hommes en armures, sans armes contre ceux portant des armes, à pied contre des cavaliers. Ils sont si agiles et féroces qu'ils gagnent souvent dans des combats fort déséquilibrés. [...] Les [...] gens du commun préfèrent se battre à pied, compte tenu du terrain marécageux et raboteux. Les cavaliers descendent souvent, si les circonstances l'exigent, prêts à fuir ou attaquer ${ }^{10}$.

En se battant, les Gallois se montraient d'abord d'une grande fermeté. En revanche, si l'ennemi venait à résister, ils étaient rapidement plongés dans la confusion.

Si la résistance qu'on leur oppose s'intensifie, les voilà qui tournent leurs dos, renonçant à toute tentative de contre-attaque, cherchant la sécurité dans la fuite. Leur courage se montre le mieux quand ils battent en retraite, car alors ils se retournent souvent, tirent leurs flèches par derrière, comme les Parthes. [...] leur seule tactique est soit de poursuivre leurs adversaires soit de s'échapper. Ils sont légèrement armés et comptent plus sur leur agilité que sur la force brute. Par conséquent, ils ne peuvent affronter leur ennemi sur un pied d'égalité, ni se battre pendant longtemps, ni chercher la victoire à la force de leur bras [...] Quoique vaincus aujourd'hui et forcés de fuir honteusement au milieu d'un grand carnage, dès le lendemain ils se remettent en route [...] Ils ne brillent peut-être pas dans le combat ouvert ou en formation fixe, mais ils harcèlent l'ennemi par leurs embuscades et leurs attaques de nuit ${ }^{11}$.

9. Lynn (2003): 12-20.

10. Giraldus (1194), I.8.

11. Giraldus (1994),II.3. 
C'est encore plus à l'Ouest, en Amérique, que les Européens rencontrèrent plus tard des peuples qui avaient encore d'autres façons de faire la guerre: ces peuples du Nouveau-Monde, les Européens avaient tout intérêt à se les représenter comme des êtres sauvages, dangereux, voire cannibales, qu'il convenait de combattre ${ }^{12}$. Cette attitude a longtemps persisté. La pratique des Indiens de l'Amérique du Nord de scalper leurs ennemis fut d'abord sentie comme particulièrement répugnante et barbare, puis petit à petit intégrée dans la pratique de guerre des «blancs». Quoique la pratique d'amputer des parties du corps de l'ennemi se retrouve en Europe, les Européens considéraient que le principe du scalp était une pratique typique des Amérindiens. Au cours des guerres «françaises et indiennes» (1754-1760), «cette coutume horrible était pratiquée par ces seuls sauvages et trouvait sa source dans leur barbarie $\gg^{13}$. Et pourtant les Européens en adoptèrent la pratique. Il leur arrivait, quand ils ne le faisaient pas eux-mêmes comme certains missionnaires chrétiens, de stipendier leurs alliés autochtones ou encore des mercenaires pour pratiquer le scalp à l'encontre de leurs ennemis communs, une pratique qui facilitait le décompte des victimes. Parce que les Européens payaient les Amérindiens pour scalper, certaines tribus à qui cette pratique était autrefois étrangère se mirent à l'adopter. Ce qui était originellement un rituel pratiqué uniquement à l'occasion de rares cérémonies se mua ainsi en pratique massive et mercantile de mutilation ${ }^{14}$.

Mais guère besoin d'aller si loin pour chercher des façons diverses de faire la guerre, et d'opposer la «nôtre» à la «leur». Cette perception dualiste persiste parmi les auteurs européens. Aux peuples nomades de l'Antiquité qui troublaient tant les Grecs et les Romains avec leurs incursions, à ceux des grandes migrations de l'Est et du Nord-Est en direction de l'ouest et du sud de l'Europe, puis aux grandes guerres de conquête des cavaliers arabes et turcs, propulsés par l'Islam, on pourrait encore ajouter les guerriers des Balkans et des grandes plaines du sud de l'Empire russe ${ }^{15}$.

L'expansion des empires coloniaux mettait les Européens en contact également avec des peuples du Sud, en Afrique. Vers la fin du XIX ${ }^{e}$ siècle, les Européens se heurtèrent à l'esprit guerrier des indigènes qu'ils commencèrent à craindre. Lors de la bataille d'Omdourman en 1898, les Britanniques armés de fusils abattirent les Soudanais, qui, courageux mais fous, «criaient comme

12. Wendt (1989).

13. J. C. B. Travels in New France, cited in Bray (1998).

14. Axtell \& Sturtevant (1980).

15. Le Mière de Corvey (1823): 1-8; 35-52. 
des démons $»^{16}$. On parlait alors du triomphe «des armes de science sur le barbarisme ${ }^{17}$.

L'attitude raciste des Européens de la haute époque du darwinisme social se reflète bien dans le célèbre manuel du Colonel britannique Charles Callwell, Small Wars (Petites Guerres, qui désignaient exclusivement les guerres coloniales), et dans les Observations sur les Guerres dans les Colonies du Lieutenant-Colonel français Alfred Ditte ${ }^{18}$. Callwell opposait la guerre civilisée en Europe à la guerre sauvage pratiquée en dehors de l'Europe. Les guerres sauvages seraient selon lui le fait de cultures singulières obéissant à des règles très spécifiques; pour venir à bout de ces sauvages, il convient, explique-t-il, de prendre des mesures hardies et résolues ${ }^{19}$. Callwell réservait une certaine admiration à la façon humanitaire avec laquelle Hoche avait tenté de maîtriser l'insurrection en Vendée dans les années 1790, mais soulignait que cette approche n'était adéquate que pour l'Europe, certainement pas pour les colonies extra-européennes ${ }^{20}$. Callwell était ainsi d'avis qu'il fallait renoncer à toute limite en combattant les peuples colonisés. À l'égard des «races noncivilisés» on confondait, selon lui, la «clémence» et la «timidité»; son verdict était que «des fanatiques et des sauvages doivent être rigoureusement soumis et effrayés, sans quoi ils se soulèveront à nouveau» ${ }^{21}$. De tels arguments, Callwell n'était pas le seul à les avancer, mais ses formules sont typiques de l'impérialisme européen de l'époque. C'est ainsi qu'un argument philosophique de l'Antiquité portant sur la nature humaine fut transformé, par l'introduction d'une polarité entre deux modes de guerre, en article de foi de la pensée militaire ${ }^{22}$. Le livre de Callwell fournissait ainsi aux militaires une rationalisation permissive de l'utilisation de la force disproportionnée contre un ennemi perçu comme inférieur et sauvage.

Les différences culturelles existent évidemment, et la perception de telles différences se remarque sans conteste dans les écrits d'anthropologues. Ce qui est remarquable, pourtant, c'est que la façon de faire la guerre en Occident pendant l'ère napoléonienne, qui avait alors valeur de paradigme ${ }^{23}$, avec une logique et une raison d'État clausewitzienne, - c'est que cette façon, disons-

16. National Army Museum (NAM), Ref. 8305-55, Captain Neville Cameron, Letter 4 Sept. 1898.

17. Churchill (1951): 300 .

18. Calwell (1896); Ditte (1905).

19. Callwell (1896): 78.

20. Callwell (1906) : 41 .

21. Ibid.: 148 .

22. Reid (2007): 15-16.

23. Voir Heuser (2013) : 95-151. 
nous, est devenue pour beaucoup d'auteurs le critère même de la modernité ${ }^{24}$. Les anthropologues Richard Schultz et Andrea Drew ont commenté le choc des cultures entre l'Occident moderne (les États-Unis d'Amérique) et le monde pré-moderne, lorsque, en 1993, les Américains échouèrent dans leur tentative de pacification en Somalie et y déclenchèrent à la place une vendetta tribale. Schultz et Drew tenaient les Américains pour prisonniers mentaux du paradigme clausewitzien, c'est-à-dire d'un modèle de guerres menées exclusivement entre États et visant exclusivement la victoire militaire, inapplicable dans un contexte extérieur à l'Occident: «La guerre tribale traditionnelle... est une guerre diffuse et très différente de sa contrepartie moderne en Occident. Elle ne reflète pas le paradigme clausewitzien ni les limitations grotiennes ${ }^{25}$. Les chefs de tribus ou de clans ne se servent pas de la guerre comme d'un outil que l'on manipule avec une froide rationalité en fonction d'un calcul imposé par une stratégie étatique; elle est au contraire menée sous l'empire de désirs socio-psychologiques, y inclus la conquête, le prestige, la satisfaction de l'ego, l'honneur, la gloire, la revanche, le vengeance et la vendetta ${ }^{26}$.»

Selon Schultz et Drew, au terme de la décolonisation, les sociétés postcoloniales seraient donc revenues à une façon «traditionnelle» de faire la guerre tandis que cette «altérité » se serait prêtée aux stéréotypes les plus simplistes. Autrement dit, tandis que les États occidentaux font la guerre dans des buts politiques, les «autres», acteurs non-étatiques, la font en poursuivant des désirs primaires. Nous nous trouvons dans des débats sur le choc des cultures, entre Européens et Amérindiens, Mexicains ou Hawaiiens, entre des autochtones dominés par des rituels primitifs, immobiles et pétrifiés dans le temps, créatures de mythe et de superstition, et les conquérants européens réfléchissant et combattant de manière stratégique ${ }^{27}$.

En attribuant à l'Occident une façon de faire la guerre fondée sur une logique clausewitzienne et grotienne, nous nous trouvons face à deux interprétations possibles: selon la première, nous ferions la guerre selon des règles internationales, d'une manière juste, tandis que l'abandon du modèle clausewitzien ou grotien mène aux sauvageries, aux atrocités et aux massacres. Après le 11 septembre, feu l'historien militaire Sir John Keegan écrivait que «la guerre contre le terrorisme» faisait partie d'un «conflit plus ancien entre les Occidentaux bien établis, créatifs, productifs, et des Orientaux prédateurs

24. Bunker (1995) : 34-41, esp. 37.

25. Limitations qui imposent à la guerre entre Etats les règles du droit de la guerre (jus ad bellum et jus in bello).

26. Shultz \& Dew (2006) : 5-6, 4, 26

27. Voir le débat entre «Sahlins et Obeyesekere», Obeyesekere (1992); et Todorov (1984) 
et destructifs.» Alors que les Occidentaux se battent honorablement «dans des batailles rangées» avec des «codes d'honneur», les Orientaux préfèrent «l'embuscade, la trahison, la tromperie ${ }^{28}$. L'historien militaire Philip Sabin (expert sur la Grèce antique) pense pour sa part que, dans un monde en défaut de clarté et de simplicité, où la guerre est soumise à un continuum globalisé de terreur, de bombardements à longue distance et de massacres de civils non-armés, nous éprouvons une sorte de regret pour les combats rangés sur des champs de bataille larges et ouverts. Il se figure que les Américains, les Britanniques et les Israéliens aspirent avec nostalgie au temps où leurs ennemis partageaient leur propre préférence pour une guerre conduite selon les règles ${ }^{29}$.

L'autre interprétation est en forme d'autocritique: l'Occident aurait développé une fixation sur le paradigme napoléonien (ou clausewitzien), mauvaise à tous égards, menant à un nombre considérable de morts. Cette fixation sur le modèle de guerres menées exclusivement entre États (alors que la majorité des conflits sanglants aujourd'hui possède une dimension intra- ou sub-étatique), jointe à l'inflexibilité manifestée dans les pourparlers, aboutissant à des conditions de paix imposées et non-négociées, ont souvent favorisé la reprise des hostilités (on pense aux conditions de paix imposées à la France en 1871 ou à l'Allemagne en 1919) ${ }^{30}$. Cette autocritique a mené en Occident à la grande (re)découverte de L'Art de la guerre de Sun-tseu ${ }^{31}$ dans les années 1980, un ouvrage qui après l'échec américain au Vietnam semblait indiquer une alternative plus souple au modèle classique de la guerre développé en Occident.

Comme nous l'avons déjà souligné, la manière occidentale de faire la guerre a en réalité considérablement varié à travers le temps et l'espace. Il y a une longue tradition occidentale consistant à éviter la bataille, à préférer l'approche indirecte, comme le remarquait déjà Sir Basil Liddell Hart durant l'entre-deux guerres ${ }^{32}$. Même les hoplites grecs recouraient à la tromperie et à la ruse quand elles servaient leur cause ${ }^{33}$. Si aux $\mathrm{VII}^{\mathrm{e}}$ et $\mathrm{VI}^{\mathrm{e}}$ siècles avant $\mathrm{J}$.C., les cités grecques avaient bien favorisé dans leur méthodes agonistiques la confrontation directe entre phalanges en plein jour selon des conventions et des règles précises, il leur arrivait toutefois de préférer les embuscades

28. Keegan (2001).

29. Cité dans Sabin (2007): xi, 29.

30. Heuser (2013): 95-151; Lynn (2003): 319.

31. Sur ce point je renvoie aux pénétrantes réflexions sur la stratégie militaire chinoise livrées par Albert Galvany dans ce volume.

32. Liddell Hart (1933)

33. Holeindre (2010). 
nocturnes, les razzias et les expédients rusés. La rhétorique politique glorifiait l'amour de l'hoplite pour les batailles sanglantes et décisives, mais que nous dit cette rhétorique de la réalité historique ${ }^{34}$ ? Dans leurs Histoires, Hérodote, Thucydide et Polybe incluaient des discours dénonçant la tromperie : est-ce là l'indice que les Grecs n'y avaient pas recours, ou simplement un moyen de les exhorter à n'en pas faire usage, en faisant appel à leur sens de l'honneur par le truchement d'un passé mythique reconstruit pour la cause ? Ces trois historiens grecs montrent aussi que les commandants recouraient à la duplicité, faisant montre d'amitié quand ils étaient animés de dispositions contraires, envoyant de fausses informations, multipliant les feintes, concluant des accords avec une ambiguïté calculé, s'attaquant à des villes non-défendues ${ }^{35}$. Pour la guerre du Péloponnèse, Krentz identifie trente-sept cas d'attaques basées sur la tromperie, la ruse ou la surprise, et seulement deux batailles rangées de fantassins - Délium et Martinea ${ }^{36}$. Les Spartiates préparaient leurs garçons à tendre des embuscades et à pratiquer l'espionnage. La culture grecque admirait Ulysse, en opposant son esprit de ruse (sa metis) avec la brutalité physique d'un Achille ${ }^{37}$. Le spartiate Brasidas se moquait des Barbares qui évitaient les batailles rangées, mais louait en même temps l'opportunisme de préférence à l'attaque directe ${ }^{38}$. Comme le font les États aujourd'hui, les Athéniens et les Spartiates dénonçaient les irrégularités commises par autrui, tout en se vantant de se situer du côté de la raison ${ }^{39}$.

Dans les guerres grecques contre les non-Hellènes, les ennemis asiatiques n'avaient pas le monopole des stratagèmes. La guerre mythique de Troie se termina avec les Danéens - les Grecs du Péloponnèse - recourant à la ruse du cheval contre les Troyens. La guerre réelle entre Athéniens et Perses aboutit à la bataille navale de Salamine, où Thémistocle attira les bateaux perses dans un piège pour donner l'avantage aux navires athéniens. Il envoya un message à Xerxès selon lequel les Athéniens étaient en train de s'enfuir, et le poussa ainsi à livrer l'une des batailles les plus décisives de l'histoire. Une telle victoire rendit possible la grande floraison culturelle d'Athènes au v'siècle ${ }^{40}$. L'Occident démocratique dépendait, lui aussi, de la ruse et de l'astuce.

34. Un argument est que le modèle agoniste disparaissait en réalité petit à petit, quoiqu'il continuait de dominer la théorie et la littérature: Pritchett (1971): 186-7; voir aussi Hanson (2000).

35. Montagu (2006): 67-81.

36. Krentz (2000)

37. Kagan (2003) : 27-42; Wheeler (1988) : xiv.

38. Krentz (2000): 174.

39. Ladis (2003).

40. Strauss (2005) : 171-253. 
Il en va de même pour les Romains. Le consul et commandant Fabius Cunctator («le temporisateur») obtint son sobriquet par sa tendance à hésiter à s'engager dans la bataille contre les Carthaginois sous Hannibal. Pourtant, les Romains aussi souscrivaient au mythe des grandes batailles. Polybe nous raconte que les «vieux sénateurs » insistaient sur le fait que la tradition romaine exigeait des batailles honnêtes et rangées. Leurs ancêtres s'étaient battus sans feintes, sans embuscades ni attaques nocturnes, refusant la fourberie «de l'astuce punique et de la finesse grecque», démontrant ainsi un authentique courage. Ils déclaraient la guerre avant de livrer bataille et annonçaient à l'avance où auraient lieu les hostilités ${ }^{41}$. D'autres propagandistes romains opposaient le courage romain à l'attitude déshonorante de leurs adversaires. Mais comme l'histoire grecque, l'histoire romaine accuse des contradictions entre rhétorique et pratique. En parlant de leurs propres ruses, les Romains les appelaient fièrement des «stratagèmes ». César dédaignait les astuces des Germains et des Gaulois, mais vantait les siennes. Historien et moraliste, Valère Maxime faisait l'éloge des stratagèmes romains comme moyen psychologiquement habile pour confondre l'ennemi, mais condamnait les manœuvres d'Hannibal à Cannes comme injustes et contraires aux règles ${ }^{42}$.

Au Moyen Âge nous trouvons des distinctions fort subtiles entre ruses autorisées et ruses condamnables. L'historien David Whetham croit y avoir décelé un modèle récurrent, mais aux yeux du non-expert, il semble plutôt que soit tout simplement défini comme mauvais ce que fait l'adversaire et comme admirable d'intelligence les actions qui relèvent de son propre camp ${ }^{43}$. En tout état de cause, Machiavel pensait qu'à l'époque antérieure à la sienne, les preux chevaliers avaient moins tendance à recourir à la ruse que dans l'Antiquité, dans la mesure où les nobles se voyaient restreints dans leurs faits et gestes par le code de chevalerie ainsi que par la considération des liens unissant les Chrétiens. En conséquence de quoi, le chef d'armée de l'époque médiévale était, toujours selon Machiavel, particulièrement vulnérable aux ruses et aux duperies ${ }^{44}$. Il y avait pourtant bien peu d'esprit chevaleresque ou chrétien dans les charges à cheval de l'époque médiévale, qui ressemblaient plus qu'à tout autre chose aux raids des Huns ou des Avars, ou encore aux razzias connues dans le monde arabe.

Si l'ère moderne, affranchie de telles inhibitions sur le plan du discours, donna naissance à l'adage «all is fair in love and war», dans la langue de la

41. Polybe 42.47.4-9.

42. Wheeler (1988): 15-16, 24, 56.

43. Whetham (2009).

44. Machiavel cité dans Whetham (2009): 7. 
perfide Albion, c'est surtout la France qui se prévalait de ses renversements d'alliances, trahissant ses voisins chrétiens au XVI siècle par une entente avec le Sultan ottoman. De tels moyens n'étaient pas, au demeurant, la prérogative du vieux monde: au cours de la guerre d'indépendance américaine, le général George Washington se faisait appeler «Fabien», parce qu'il évitait autant que possible, à l'image du consul et chef de guerre romain Fabius Cunctator - le « temporisateur» - la bataille avec les Britanniques. Même à l'apogée de l'ère du paradigme napoléonien, pendant la Guerre Civile américaine, le général sudiste Thomas «Stonewall» Jackson répétait à ses troupes, «Mystifiez l'ennemi, n'ayez de cesse de le tromper!» ${ }^{45}$ En 1944, en pleine guerre mondiale, le général britannique William Slim eut les mêmes mots pour ses soldats qui se battaient contre les Japonais ${ }^{46}$. Plus l'ennemi était craint et plus apparaissait indésirable l'idée d'une bataille décisive pour emporter la victoire. Si bien que devenait tout à fait acceptable le recours à des moyens irréguliers, rejoignant par là une longue tradition d'expédients devenus nécessaires dans un contexte stratégique, que ce fût contre Hannibal au terme du carnage de la bataille de Cannes, contre l'Union du Nord dotée de toute la force de son industrie, ou encore contre l'armée japonaise au cœur de la jungle birmane. Comme même Clausewitz le faisait observer:

Plus sont faibles les forces soumises à la direction stratégique, plus celle-ci sera accessible à la ruse. Si bien que celui qui est très faible, tout petit, et à qui la prudence et la sagesse ne servent plus à rien, en vient au point où toutes les ressources de l'art semblent abandonner et où la ruse est son dernier recours. Plus sa situation est désolée, plus tout le pousse à tenter un dernier coup désespéré, et d'autant plus volontiers la ruse s'allie à l'audace ${ }^{47}$.

Tout l'objet de cette étude a été en un sens de faire mesurer au lecteur combien cette analyse que livre Clausewitz du recours à la ruse et à d'autres expédients censés caractériser la façon de livrer le combat en Orient, constituent en réalité une caractéristique commune à toutes les cultures à un moment donné de leur histoire.

45. D'Aoust (2006): 42-50.

46. BDA JSCSC 2751, «Japanese Ruses, issued by the GSIHQ Fourteenth Army March 1944.» Slim encouragea ses effectifs à être plus imaginatifs que les Japonais dans leurs ruses: «Ne vous contentez pas de rire de ses ruses maladroites, mais imaginez-en de bonnes vous-mêmes et gagnez sur lui.»

47. Clausewitz (1832): III.10. 


\section{Bibliographie}

Axtell James \& William C. Sturtevant (1980). «The Unkindest Cut, or Who Invented Scalping». The William and Mary Quarterly 31 : 3, July: 451-472, 461-2.

BRAY George A. (1998). «Scalping during the French and Indian War». Early America Review 2:3 (Spring/Summer), http://www.earlyamerica.com/review/1998/ scalping.html accédé le 2 VI 2014.

Bunker Robert J. (1995). «Rethinking OOTW». Military Review (Nov.-Dec.) : 34-41

Callwell Charles E. (1896). Small Wars: Their Principles and Practice. Londres.

Callwell Charles E. (1906). Small Wars, Their Principles and Practice. $3^{\text {rd }}$ edn, Londres.

Clausewitz Carl von (1832). De la Guerre traduit par Denise Naville. Paris, Éditions de Minuit, 1955.

Churchill Winston (1951). The River War : an Account of the Reconquest of the Sudan. Londres.

D’Aoust Maurice (2006). «Hoodwinked During America's Civil War: Confederate Military Deception». Civil War Times (June) : 42-50.

DitTÉ Alfred (1905). Observations sur les guerres dans les colonies. Paris.

ELLIS John (1975). A Short History of Guerrilla Warfare. Londres.

Eschyle (472 BCE). Les Perses.

GIRALDus Cambrensis (1194). Descriptio Cambriae

GoldhILl Simon (1988). «Battle Narrative and Politics in Aeschylus ».Persae’, Journal of Hellenic Studies, 108: 189-93.

HAHLweg Werner (1968). Guerilla: Krieg ohne Fronten. Stuttgart.

Hanson Victor Davis (1989). The Western Way of War: Infantry Battle in Classical Greece. New York, Alfred Knopf.

Hanson Victor Davis (2000). «Hoplite Battle as Ancient Greek Warfare: When, Where and Why?». In Hans van Wees (dir.) War and Violence in Ancient Greece. Londres : 201-232.

HeusER Beatrice (1998). Nuclear Mentalities? Strategies and Belief Systems in Britain, France and the FRG. Londres.

Heuser Béatrice (2013). Penser la Stratégie. Paris.

Holeindre Jean-Vincent (2010). «Le renard et le lion. La ruse et la force dans le discours de la guerre». PhD diss., École des Hautes Études en Sciences Sociales.

Kagan Robert (2003). Paradise and Power: America and Europe in the New World Order. Londres.

KEEGAN John (2001). «In this war of Civilisations, the West will prevail». Daily Telegraph, 8 Oct.

Krentz Peter (2000). «Deception in Archaic and Classical Greek Warfare». In Hans van Wees (dir.), War and Violence in Ancient Greece. Londres: 167-200.

LADIS Nikolaos (2003). «Assessing Greek Strategic Thought and Practice: Insights from the Strategic Culture Approach», doctoral dissertation. University of Southampton. 
LAQUEUR Walter (1977). Guerrilla Reader : A Historical and Critical Study. Londres. Le Mière de Corvey J.F.A. (1823). Des Partisans et des corps irréguliers. Paris.

LidDELl Hart Basil (1933). The Ghost of Napoleon: The Movement of Military Thought from the 18th to the 20th Century and Its Influence on European History. Réimpression, Londres.

LuCAIN (av. 65 CE). Pharsalia.

Lynn John A. (2003). Battle : A History of Combat and Culture. Boulder, Co.: Westview. Montagu John Drogo (2006). Greek and Roman Warfare. Battles, Tactics and Trickery. Londres.

OBEYESEKERE Ganath (1992). The Apotheosis of Captain Cook: European Mythmaking in the Pacific. Princeton, NJ : Princeton UP.

PORTER Patrick (2009). Military Orientalism: Eastern War Through Western Eyes. Londres.

PritchetT William K. (1971). The Greek State at War. Berkeley.

ReID Richard J. (2007). «Revisiting Primitive War: Perceptions of Violence and Race in History ». War and Society,26: 2 (Oct.) : 1-25.

Rhodes P. J. (2007). «The Impact of the Persian Wars on Classical Greece». In Emma Bridges, Edith Hall and P. J. Rhodes (dir.),Cultural Responses to the Persian Wars : Antiquity to the Third Millennium. Oxford: 31-47.

SABIN Philip (2007). Lost Battles: Reconstructing the Great Clashes of the Ancient World. Londres.

Shultz Jr. Richard H. and Andrea J. Dew (2006). Insurgents, Terrorists and Militias : The Warriors of Contemporary Combat. New York.

Strauss, Barry (2005). Salamis: The Greatest Naval Battle of the Ancient World, 480 $B C$. Londres.

Todorov Tzvetan (1984). The Conquest of America: The Question of the Other. Trans. Richard Howard, New York.

WendT Astrid (1989). Kannibalismus in Brasilien: eine Analyse europäischer Reiseberichte und Amerika-Darstellungen für die Zeit zwischen 1500 und 1654. Frankfurt/Main.

WHEELER Everett (1988). Stratagem and the Vocabulary of Military Trickery. Leiden.

Wheтнам David (2009). Just Wars and Moral Victories: Surprise, Deception and the Normative Framework of European War in the Later Middle Ages. Leyden. 